\title{
Implementación de recursos audiovisuales como estrategia de validación pedagógica*
}

\author{
Julián Darío Forero Sandoval ${ }^{\star \star}$ \\ Estela Díaz Buitrago ***
}

Recibido: 2017-09-21. Enviado a pares: 2017-09-30.

Aprobado por pares: 2017-10-25. Aceptado: 2017-11-11

https://doi.org/10.22395/angr.v17n33a12

\begin{abstract}
Resumen
El presente artículo es producto de una investigación con diseño cuasi-experimental, cuyo objetivo fue validar una estrategia pedagógica para implementar recursos didácticos audiovisuales en instituciones educativas. Su fundamentación teórica se configuró a través de dos variables: las prácticas didácticas (variable dependiente) y los recursos audiovisuales (variable independiente). Con un modelo de grupo control de grado 11 de la Institución Educativa Camilo Torres, de Mocarí (Montería, Colombia), se lograron identificar las necesidades de aprendizaje más frecuentes que presentan los estudiantes de básica secundaria, clasificar los recursos didácticos audiovisuales con que cuentan los docentes y modelar, implementar y validar una estrategia pedagógica a través del montaje de tres productos audiovisuales, tres programas de radio y un blog.

Palabras clave: recursos audiovisuales, didáctica, estrategias pedagógicas, necesidades de aprendizaje.
\end{abstract}

El artículo es resultado de la investigación Validación de una estrategia pedagógica para la implementación de recursos didácticos audiovisuales en instituciones educativas en Montería, financiado por la Universidad Pontificia Bolivariana (Radicado CIDI 170-01/15-G015). Grupo de Investigación Coedu, línea Comunicación y educación.

** Comunicador social-periodista, magíster en Educación, integrante del grupo Coedu. Escuela de Ciencias Sociales y Humanas, Universidad Pontificia Bolivariana. julian.forero@upb.edu.co Orcid: 0000-0002-5283-8425.

*** Licenciada en Informática Educativa y Medios Audiovisuales, magíster en Educación, docente, coordinadora académica de la Institución Educativa José María Córdoba, Montería. estela_mireya@hotmail.com Orcid: 0000-0002-57930298 


\title{
Implementation of audiovisual resources as an educational validation strategy
}

\begin{abstract}
This paper is the outcome of a quasi-experimental research, whose objective was to validate an educational strategy aimed at implementing audiovisual learning resources in educational institutions. Its theoretical foundation was configured through two variables: educational practices (dependent variable), and audiovisual resources (independent variable). With a control group model comprised of 11 th grade students from the Institución Educativa Camilo Torres of Mocarí (Montería, Colombia), it was possible to identify the most frequent learning needs of secondary education students, to classify the audiovisual educational resources available to the teachers, and to model, implement and validate an educational strategy by assembling three audiovisual products, three radio programs, and a blog.
\end{abstract}

Keywords: Audiovisual resources, teaching, educational strategies, learning needs.

\section{Implementação de recursos audiovisuais como estratégia de validação pedagógica}

\begin{abstract}
Resumo
O presente artigo é produto de uma pesquisa com desenho quase experimental, cujo objetivo foi validar uma estratégia pedagógica para implementar recursos didáticos audiovisuais em instituições educativas. Sua fundamentação teórica configurou-se por meio de duas variáveis: as práticas didáticas (variável dependente) e os recursos audiovisuais (variável independente). Com um modelo de grupo controle do $11^{\circ}$ ano da Instituição Educativa Camilo Torres, de Mocarí (Montería, Colômbia), conseguimos identificar as necessidades de aprendizagem mais frequentes que os estudantes de secundária apresentam, classificar os recursos didáticos audiovisuais com os quais contam os docentes e modelar, implementar e validar uma estratégia pedagógica por meio da elaboração de três produtos audiovisuais, três programas de rádio e um blog.
\end{abstract}

Palavras-chave: recursos audiovisuais, didática, estratégias pedagógicas, necessidades de aprendizagem. 


\section{Introducción}

La génesis de esta investigación está en los cuestionamientos inherentes a la relación comunicación y educación en el contexto del aula de clases que discurre en horizontes tan diversos y complejos que pretender hacerlo desde la interdisciplinariedad no es una tarea de corto aliento. Sustentado en este supuesto epistemológico, se ha planteado como objetivo de la investigación validar una estrategia pedagógica que permita implementar recursos didácticos audiovisuales en instituciones educativas de Montería (Departamento de Córdoba, Colombia) bajo una pretensión educativa que se instaura en los postulados de la pedagogía liberadora:

El diálogo y la problematización no adormecen a nadie. Concientizan. En la dialoguicidad, en la problematización, educador-educando y educando-educador desarrollan ambos una postura crítica, de la cual resulta la percepción de que todo este conjunto de saber se encuentra en interacción. Saber que refleja al mundo y a los hombres, en el mundo y con él, explicando al mundo, sobre todo justificándose en su transformación. (Freire, 1973, p. 62).

Diálogo que desde la concepción freireana no se ha explicitado en el micromundo llamado aula de clases, donde se representa y simboliza el conocimiento con todos sus entramados e intersticios que, se supone, median el proceso de enseñanza-aprendizaje y que en la tradición de la escuela de Occidente se ha soportado en ayudas que permiten exponer los saberes, pero que en la gran mayoría de los casos, particularmente cuando se usan medios audiovisuales, no superan las posibilidades técnico-instrumentales de almacenar y transmitir mensajes codificados visual y sonoramente.

Es en este ámbito en el que los medios audiovisuales y las TIC han llegado al mundo educativo. En la gran mayoría de las ocasiones, bajo presiones ajenas a la institución escolar, asociadas más con el fenómeno de la sociedad de consumo que a principios fundantes de la pedagogía y la educación. Así como lo señaló Mallas (1979) hace más de 30 años, este proceso de penetración no ha permitido a los educadores e investigadores de la educación un análisis profundo del tema, sino que se han incorporado al proceso de enseñanza-aprendizaje, motivado por el frenesí de la ocasión y sin reflexionar sobre qué podría esperarse de estos recursos, cómo debían organizarse y cuáles eran los más convenientes a utilizar. Resultado de ello, en muchos ambientes, se generó rechazo e incluso hostilidad hacia estos medios-recursos. Hostilidad que se le endosa al advenimiento de lo que Porcher (1974) denomina la élite irresponsable, que funciona como antagonista de los docentes al usurparles una de sus funciones esenciales: ser mediador entre la cultura y la transmisión del conocimiento.

Esta primera mirada permite identificar que, en el aula de clase, se debe superar el uso instrumental de los medios, para ser comprendidos más allá de sus características técnicas. No es suficiente con la alfabetización tecnológica que trae consigo la llegada de un nuevo recurso audiovisual. Es concurrente la tendencia a confundir el manejo del aparato con las formas expresivas que el mismo ofrece, poniendo en el mismo nivel la acumulación y distribución de información con la construcción de conocimiento. 
Si por las circunstancias que fuere, se plantea la utilización en la educación de los medios en general, o de las nuevas tecnologías en la educación, sin solucionar como es debido sus dimensiones sustantivas y valorativas, estaríamos apostando probablemente, por una posible innovación técnica, no por una innovación educativa. Si, de otro lado, conseguimos asentar debidamente cuestiones tales como las referidas a las metas y propósitos educativos para el uso de las nuevas tecnologías, cuándo y a qué alumnos, en qué tipo de contenidos y en cómo trabajarlos en el aula, pero descuidamos los cómo, las condiciones y los procesos a través de los que realizar las innovaciones de este tipo en contextos y circunstancias particulares, correríamos todos los riesgos de practicar una política de buenas intenciones y declaraciones con escasas posibilidades de desarrollarse en la práctica de los centros y el quehacer cotidiano de los profesores y los alumnos. Es por eso que estas tres cuestiones generales, pese a ser diferenciadas analíticamente, han de ser integradas en nuestro pensamiento y praxis pedagógica en relación con la integración de las nuevas tecnologías en la educación. (Escudero, 1995, p.405).

Desde este horizonte, la investigación buscó trabajar la intención comunicativa que los medios audiovisuales (productos sonoros, videos, multimedia, etc.) ofrecen como recursos para la validación de una estrategia pedagógica, teniendo en cuenta los siguientes supuestos:

- Es prioritaria la selección de la información que es de uso exclusivo en el diseño del medio audiovisual y la qué es potestad del docente. Esta priorización obedece a una intención comunicativa que debe articular y dar respuesta a las necesidades e intereses de la comunidad académica, producto del trabajo entre docentes, estudiantes del área y los profesionales responsables del diseño y montaje del recurso didáctico.

- Son las nuevas narrativas que cada recurso audiovisual ofrece como expresión del lenguaje las que median en la construcción del conocimiento.

- Una adecuada planeación lleva a definir con tiempo cómo usar el recurso didáctico escolar de manera asertiva en cada contexto escolar.

- La implementación de cada medio audiovisual como recurso didáctico, debe hacer adaptables sus valores implícitos con los valores que predominan en el contexto escolar.

Bajo este prisma educativo, se comprende entonces, como lo afirma Marrero (1993), al docente como mediador entre el estudiante y la cultura, por el sentido que arraiga el currículo en general y por el conocimiento que de allí se transmite en particular: "la tamización del currículum por los profesores no es un mero problema de interpretaciones pedagógicas diversas, sino también de sesgos en esos significados que, desde un punto de vista social, no son equivalentes ni neutros" (Marrero 1993, p. 243). 
Aunque en teoría se plantea un desplazamiento del centro de interés desde el maestro hacia el alumno, es difícil afirmar que esto ocurra siempre en la práctica (...).

La meta de los nuevos ambientes de aprendizaje es crear comunidades de conocimiento y, aunque con frecuencia se basan en un mejor aprovechamiento de los últimos adelantos tecnológicos, para operar el cambio social su base teórica y conceptual debe estar en el vínculo comunicación-educación. Por ello, es responsabilidad de los actores del proceso educativo y de los investigadores seguir insistiendo en que las tecnologías son sólo un factor de mediación, un apoyo para lograr el propósito de una mejor educación que nos permita aprender el arte de vivir en un mundo saturado de información. (Crovi 2010, p. 120).

Se comprende así que la adopción de recursos en el aula no es un asunto producto del azar o de las buenas intenciones de un docente. Blázquez (1994) propone una serie de criterios que se deben considerar al momento de articular los medios audiovisuales con el currículo, a saber: la calidad de los recursos, pues su adecuación depende de la realidad escolar, los sujetos participantes y el uso particular de los códigos inherente a cada medio; los contextos metodológicos que deben estar en correspondencia con los objetivos planteados; la concreción de los destinatarios, ya que cada medio debe adaptarse a las necesidades y posibilidades de los estudiantes y, finalmente, la vehiculización del profesor, entendida como la mediación que hace el docente entre el conocimiento y cada uno de los factores decisivos a la hora de determinar el grado de asertividad del medio.

\section{Metodología}

En correspondencia con las características inherentes a la investigación y con el propósito de alcanzar los objetivos del estudio: responder al planteamiento del problema y someter a prueba los supuestos formulados, se trabajó con un diseño cuasi-experimental que se caracteriza por presentar dos grupos, uno experimental y otro de control (Hernández, Fernández y Baptista, 1999). Ver tabla 1.

Tabla 1. Esquema representativo para grupos experimental y control

\begin{tabular}{|l|c|c|c|}
\hline \multicolumn{1}{|c|}{ Grupos } & Medida de la preprueba & Tratamiento & Medida de la posprueba \\
\hline Experimental & 01 & $\mathrm{X}$ & 02 \\
\hline Control & 01 & - & 02 \\
\hline
\end{tabular}

Fuente: Díaz, Alvarino y Carrascal, 2011

Para el desarrollo del estudio se tuvieron en cuenta las siguientes variables: una dependiente que son las prácticas didácticas y una independiente, los recursos audiovisuales. Para evaluar estas variables se configuraron dimensiones e indicadores que caracterizan sus aspectos estructurales (tabla 2). 
Tabla 2. Dimensiones e indicadores de variable

\begin{tabular}{|c|c|c|c|}
\hline $\begin{array}{c}\text { Variable } \\
\text { dependiente }\end{array}$ & Definición & Dimensión & Indicadores \\
\hline $\begin{array}{l}\text { Prácticas } \\
\text { didácticas }\end{array}$ & $\begin{array}{l}\text { Actividades } \\
\text { pedagógicas } \\
\text { que median la } \\
\text { comunicación } \\
\text { entre docentes } \\
\text { y estudiantes } \\
\text { en torno a los } \\
\text { contenidosob- } \\
\text { jeto de estu- } \\
\text { dio, apoyados } \\
\text { en un recurso } \\
\text { didáctico. }\end{array}$ & $\begin{array}{l}\text { Relaciones } \\
\text { pedagógicas }\end{array}$ & $\begin{array}{l}\text { - Condiciones ambientales favorables para la } \\
\text { utilización de los recursos didácticos. } \\
\text { - Relación comunicativa entre el discurso del } \\
\text { docente y la capacidad de escucha del estu- } \\
\text { diante. } \\
\text { - Las prácticas didácticas en función de los } \\
\text { contenidos por aprender. } \\
\text { - Las actividades en el aula de clase, trabajan } \\
\text { la información de manera pedagógica. } \\
\text { - Definición de criterios pedagógicos para la } \\
\text { utilización de los recursos. } \\
\text { - Integración de los recursos didácticos en el } \\
\text { proyecto curricular. }\end{array}$ \\
\hline $\begin{array}{c}\text { Variable } \\
\text { independiente }\end{array}$ & $\begin{array}{l}\text { Definición } \\
\text { conceptual }\end{array}$ & Dimensión & Indicadores \\
\hline \multirow[b]{2}{*}{$\begin{array}{l}\text { Recursos } \\
\text { diácticos }\end{array}$} & \multirow{2}{*}{$\begin{array}{l}\text { Materiales } \\
\text { educativos } \\
\text { construido } \\
\text { con palabras, } \\
\text { sonidos e imá- } \\
\text { genes, que se } \\
\text { constituyen en } \\
\text { elementos me- } \\
\text { diadores y que } \\
\text { facilitan comu- } \\
\text { nicar los con- } \\
\text { tenidos que } \\
\text { son objeto de } \\
\text { aprendizaje. }\end{array}$} & $\begin{array}{l}\text { Lenguaje } \\
\text { audiovisual }\end{array}$ & $\begin{array}{l}\text { - El uso de los recursos audiovisuales que } \\
\text { facilita la comunicación docente-estudiante } \\
\text { con miras a su aprendizaje. } \\
\text { - Tipo de recurso audiovisual más utilizado por } \\
\text { los docentes. } \\
\text { - Tipo de recurso audiovisual que le facilita al } \\
\text { docente la preparación de clases. } \\
\text { - Lugar de uso de la ayuda } \\
\text { - Uso creativo de los recursos audiovisuales. }\end{array}$ \\
\hline & & $\begin{array}{l}\text { Mediaciones } \\
\text { comunicativas }\end{array}$ & $\begin{array}{l}\text { - El uso del recurso audiovisual ayuda como } \\
\text { práctica didáctica que fomenta la participa- } \\
\text { ción activa y crítica de los estudiantes. } \\
\text { - Reconocimiento de las posibilidades forma- } \\
\text { tivas que ofrece el recurso audiovisual. } \\
\text { - Uso contextual del recurso audiovisual. } \\
\text { - Los recursos audiovisuales, de acuerdo a sus } \\
\text { valores implícitos, evalúan su forma, estruc - } \\
\text { tura, función e impacto en el medio escolar. } \\
\text { - Concepción del recurso audiovisual a utilizar } \\
\text { de acuerdo al proceso comunicativo en el que } \\
\text { se está inmerso. }\end{array}$ \\
\hline
\end{tabular}

Fuente: Díaz et al., 2011. 


\section{Población sujeto de estudio}

La población estuvo conformada por 73 estudiantes matriculados en el año lectivo 2016 en el grado $11^{\circ}$, pertenecientes a la Institución Educativa Camilo Torres. Para el estudio, dada la distribución de los estudiantes y para poder validar la propuesta en ambos géneros, se tomó el grupo 11-3 como grupo experimental y el grupo 11-2 permaneció como grupo control. Cabe anotar que, para la validez de la investigación, se hizo necesario establecer la similitud o equivalencia de ambos grupos a partir de los criterios que se describen a continuación en la tabla 3.

Tabla 3. Equivalencia entre grupos

\begin{tabular}{|l|c|c|}
\hline \multicolumn{1}{|c|}{ Criterios } & $\begin{array}{c}\text { Gruposl1-3 } \\
\text { (Experimental) }\end{array}$ & $\begin{array}{c}\text { Grupos 11-2 } \\
\text { (Control) }\end{array}$ \\
\hline Número de estudiantes & $39(53 \%)$ & $34(47 \%)$ \\
\hline Nivel socioeconómico & Estratos 1 y 2 & 17 años \\
\hline Promedio de edad & 16 años & 8 \\
\hline Número de repitentes & 7 & Diurno \\
\hline Horario & Diurno & y 2 \\
\hline
\end{tabular}

Fuente: Díaz et al., 2011

\section{Instrumentos para la recolección de la información}

- Para la identificación de las necesidades de aprendizaje más frecuentes que presentan los estudiantes de básica secundaria y media en la I.E. Camilo Torres, se utilizaron cuestionarios aplicados que responden a tres dimensiones: relaciones pedagógicas, lenguaje audiovisual y mediaciones comunicativas. La medición se hizo a través de una escala tipo Likert de cinco puntos.

- Para clasificar los recursos didácticos audiovisuales con que cuenta la I.E. Camilo Torres, se trabajó un formato, a manera de inventario, que permitió obtener información sobre el número, tipo, características de uso e intenciones didácticas de los recursos.

- Para definir el área y los contenidos que se adaptarán a los diferentes recursos didácticos audiovisuales, se utilizó la técnica de grupo focal, donde se definió trabajar en el curso de Filosofía, el tema de la moral y la ética. 


\section{Resultados}

Los resultados que se reseñan a continuación responden a los elementos más significativos en correspondencia con los objetivos de la investigación. La primera parte de los resultados obedece a los instrumentos aplicados a los docentes de la institución educativa (ver figura 1).

El 66,6 \% de los recursos didácticos más usados corresponden a ayudas tradicionales, principalmente tablero, libros y carteleras, mientras que el uso de TIC se hace ocasionalmente, alrededor de un $33 \%$ a través de páginas web, blogs, wikis, entre otros.

En una tendencia del $75 \%$, los docentes consideran que el uso de los recursos sí fomenta la participación activa y crítica de los estudiantes (ver figura 2).

No se evidencia que los docentes conozcan de manera anticipada las posibilidades formativas que ofrece cada recurso didáctico, solo el $45,4 \%$ lo hace de forma permanente (ver figura 3).

Hay un conocimiento total y parcial del $100 \%$ de los docentes sobre las condiciones ambientales y sociales del contexto donde utilizan los diversos recursos didácticos (ver figura 4).

No hay un acuerdo generalizado que permita evidenciar cómo los docentes evalúan el impacto de los recursos didácticos en el aula de clase. Un 16,6 \% reconoce no hacerlo, en contraste con el 33,4\% que siempre lo evalúa (ver figura 5).

Los docentes reconocen total y parcialmente (100\%), las posibilidades comunicativas que ofrece cada recurso didáctico (ver figura 6).

La segunda parte de los resultados también reseña los resultados más significativos para la investigación, pero se presentan en tablas comparativas entre las respuestas de los docentes y los estudiantes de la institución educativa.

No hay un acuerdo entre docentes y estudiantes sobre la frecuencia con la que se usan los recursos didácticos. En general, no se evidencia un uso permanente de los mismos en el aula de clase. En promedio, 55,2 \% de docentes y estudiantes reconocen un uso frecuente y permanente de las ayudas en el salón de clase (figura 7).

En promedio, el 39,5\% de docentes y estudiantes considera que no hay las condiciones ambientales favorables para la utilización de los recursos didácticos. Es significativo que solo el 7,4 \% de los docentes esté totalmente de acuerdo con las condiciones ambientales de los salones de clase (ver figura 8).

Se evidencia parcial y totalmente, particularmente por parte de los docentes $(91,5 \%)$ el uso de la expresión oral como una mediación del lenguaje que favorece la relación comunicativa de los estudiantes. Para un 15,6 \% de los estudiantes, la expresión oral no favorece la relación comunicativa con sus docentes (ver figura 9). 


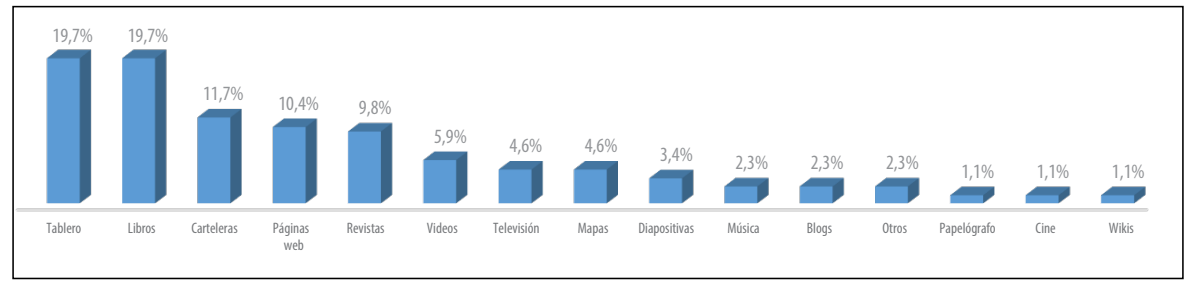

Figura 1. Recursos didácticos más usados

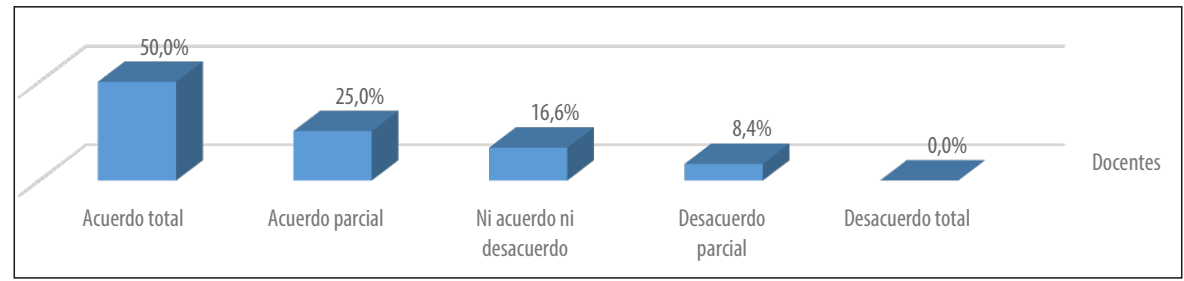

Figura 2. Uso de los recursos didácticos

que fomentan la participación activa y crítica de los estudiantes

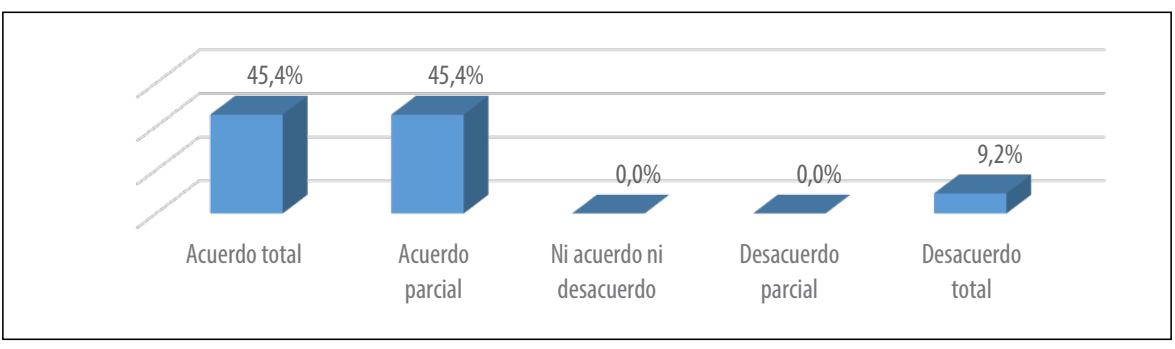

Figura 3. Conocimiento anticipado

de las posibilidades formativas que ofrece el recurso didáctico

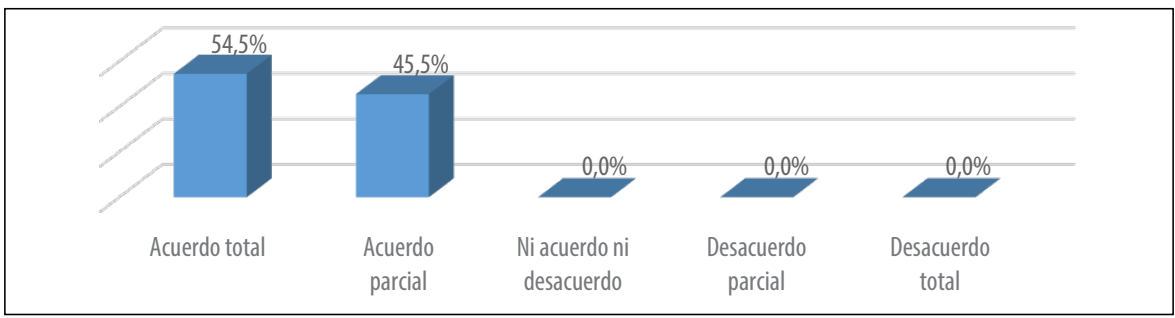

Figura 4. Reconocimiento de los contextos

en los cuales se utilizan los diferentes recursos didácticos

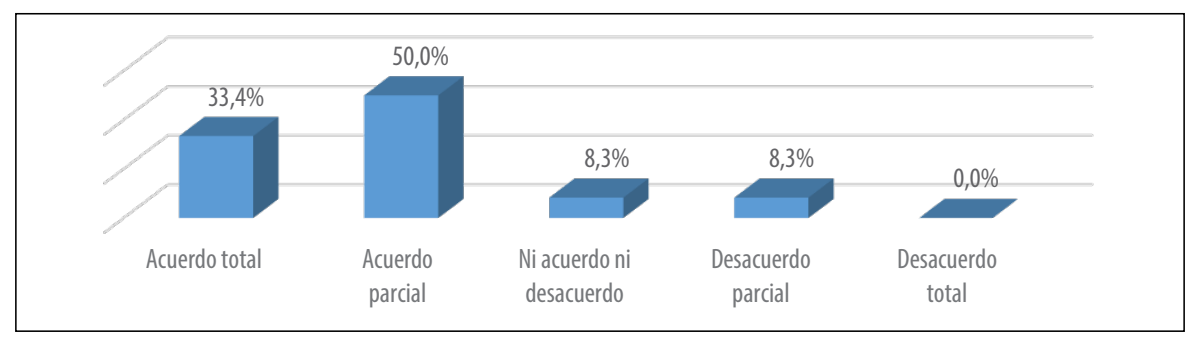

Figura 5. Evaluación del impacto de los recursos didácticos en el aula de clase 


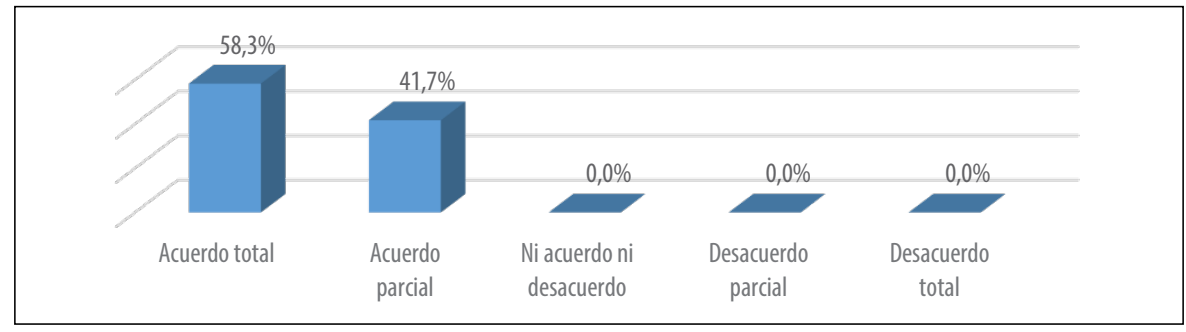

Figura 6. Definición del recurso didáctico de acuerdo a los procesos comunicativos que se dan al interior del aula de clase

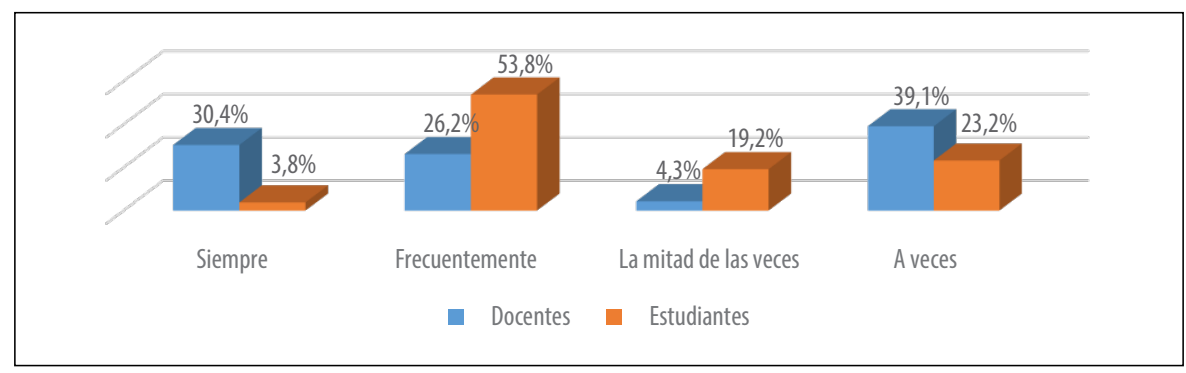

Figura 7. Frecuencia con la que los docentes usan los recursos didácticos

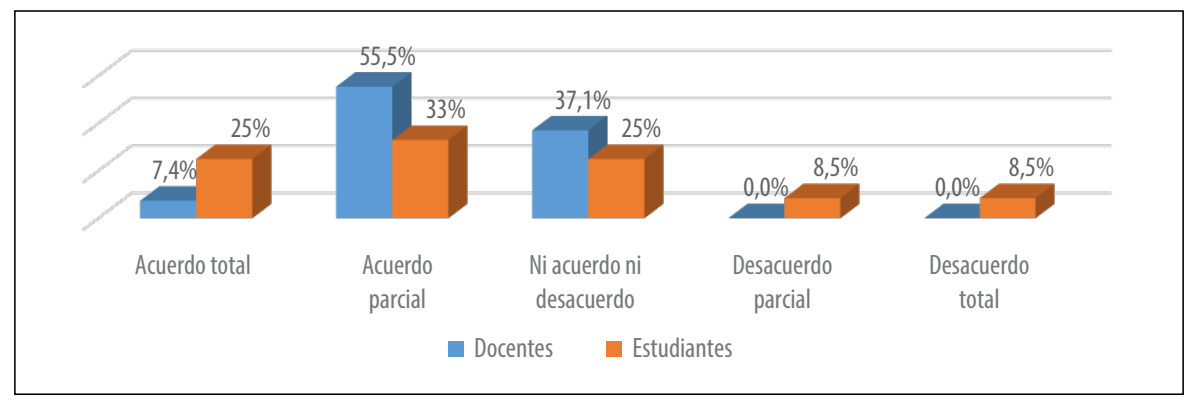

Figura 8. Condiciones ambientales favorables para la utilización de los recursos didácticos

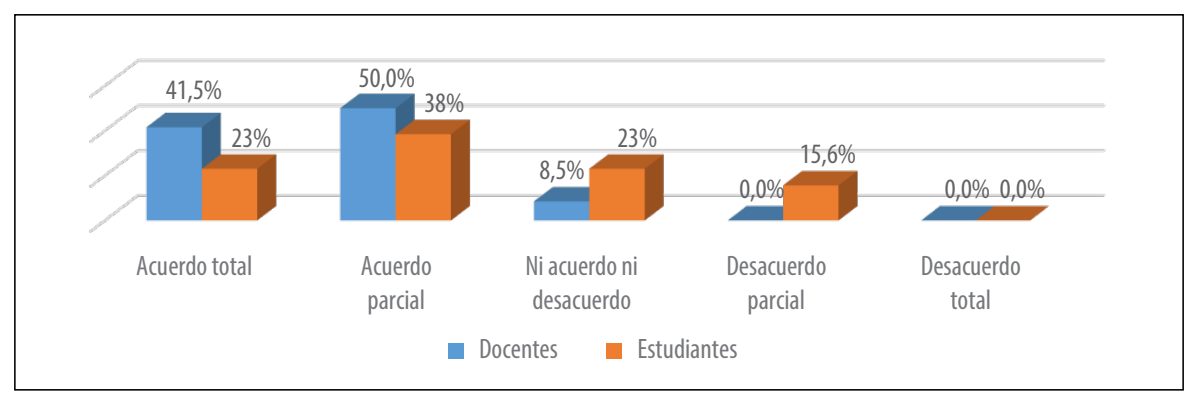

Figura 9. Uso de la expresión verbal del docente que favorece la relación comunicativa con los estudiantes 
No hay un acuerdo generalizado en torno a considerar que los recursos didácticos favorezcan la capacidad de escucha del estudiante. Los porcentajes más altos marcan la tendencia hacia un acuerdo parcial, además, un 19,3\% de los estudiantes consideran que los recursos didácticos usados por sus docentes, no tienden a estimular la capacidad de escucha (ver figura 10).

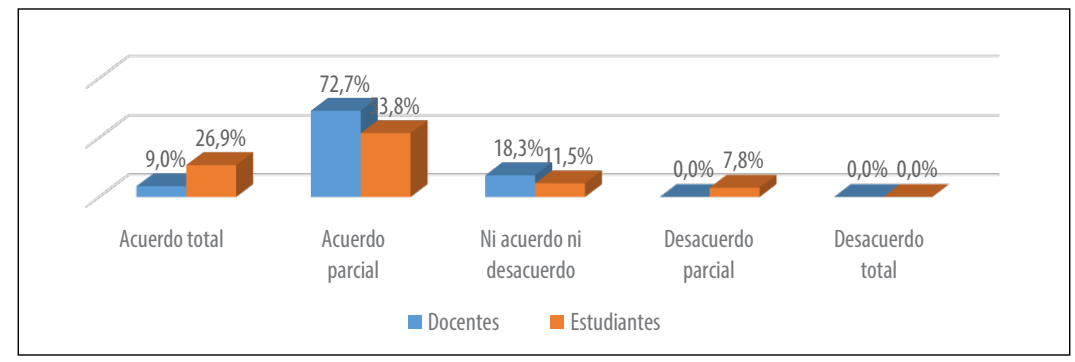

Figura 10. Capacidad de escucha del estudiante en el aula de clase

Son muy disímiles las apreciaciones entre docentes y estudiantes al considerar que las prácticas didácticas favorezcan la adquisición de los contenidos. De hecho, se da un $17,5 \%$ de estudiantes que creen que a través de dichas prácticas no se adquiere conocimiento (ver figura 11).

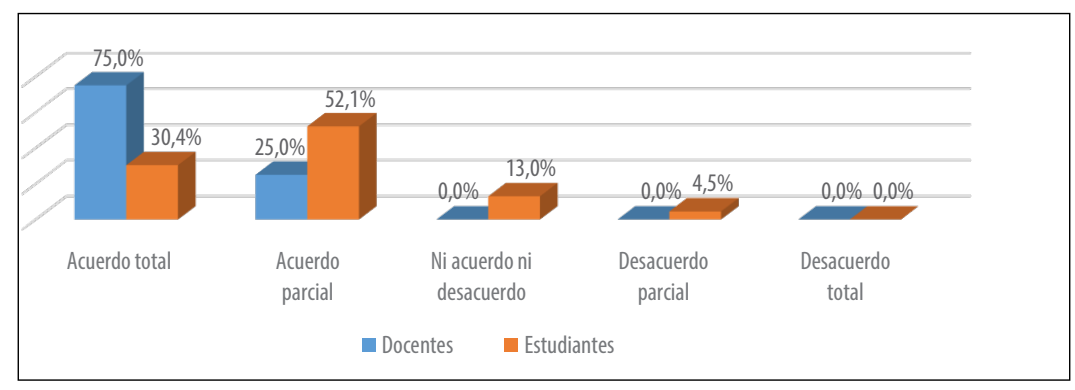

Figura 11. Prácticas didácticas que favorecen la adquisición de los contenidos a aprender

Hay un consenso general, $100 \%$ de los docentes y $84,5 \%$ de los estudiantes, que considera que la información que se les da en los cursos favorece la adquisición de conocimientos (figura 12).

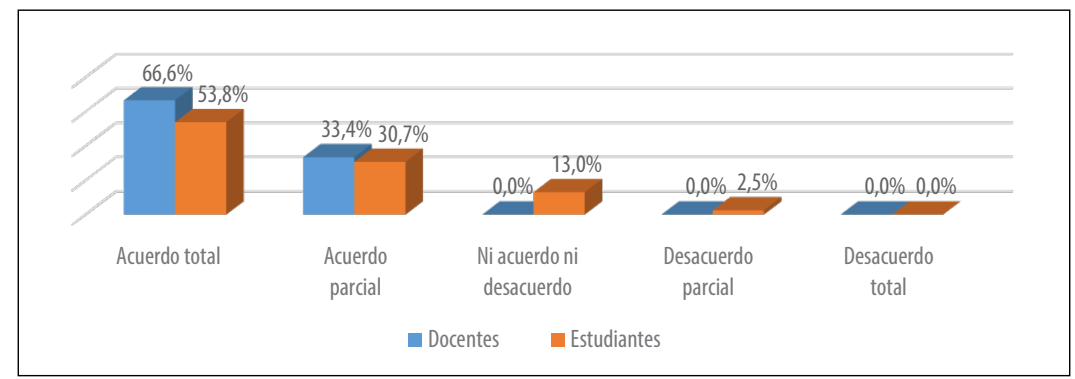

Figura 12. Desarrollo de información que favorece la adquisición del conocimiento 
Se evidencia un contraste entre docentes y estudiante en torno a si hay un conocimiento anticipado de los recursos a utilizar, pues mientras el $100 \%$ de los maestros están parcial y totalmente de acuerdo, hay un 30,8 \% de los estudiantes que consideran que no se conocen con antelación los recursos que se va a trabajar en clase (ver figura 13).

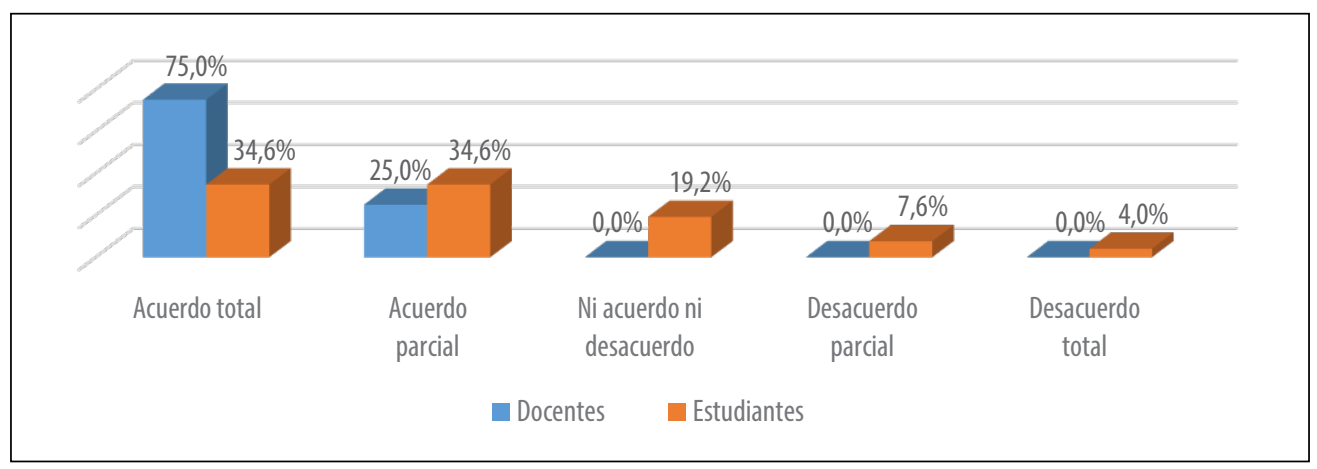

Figura 13. Conocimiento anticipado de los recursos didácticos a utilizar en el salón de clase

Se da una tendencia promedio del 85,7\% entre docentes y estudiantes que consideran creativos los recursos didácticos utilizados en el aula de clase (ver figura 14).

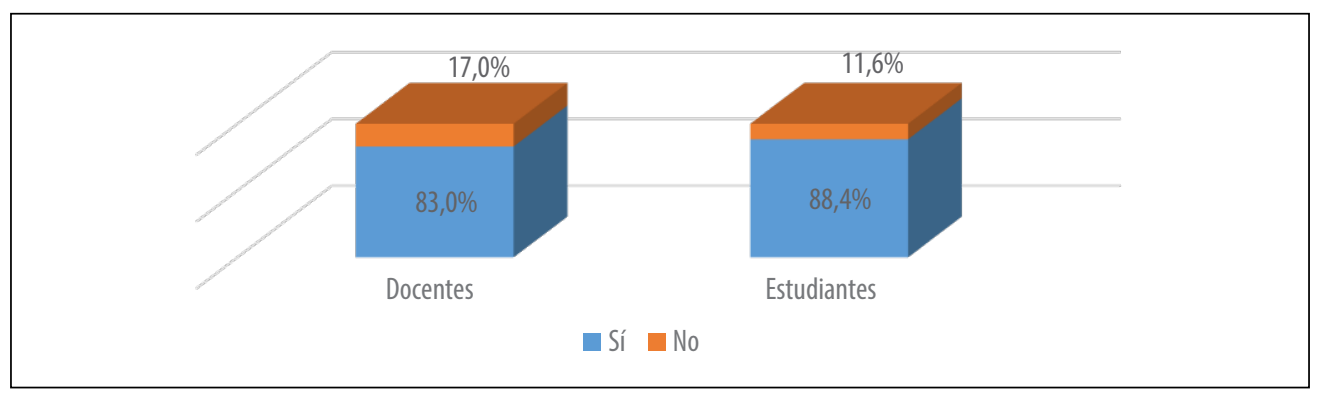

Figura 14. Uso creativo de los recursos didácticos audiovisuales utilizados por los docentes en clase

\section{Conclusiones}

Con la intención de realizar un ejercicio articulado en correspondencia con los objetivos del proyecto, se presentan las siguientes conclusiones a partir de las tres dimensiones definidas desde el marco teórico y aplicadas a través del diseño metodológico: relaciones pedagógicas, lenguaje audiovisual y mediaciones comunicativas, cada una de ellas sustentada en una fuente conceptual. 


\section{Relaciones pedagógicas}

Para las conclusiones de esta dimensión se tomará como fundamento teórico el texto Prácticas educomunicativas de Gall (2005), quien concibe la comunicación y la educación como actividades grupales, espacio para el diálogo en donde el docente, bajo un rol de comunicador establece y favorece relaciones pedagógicas, "no para transmitir un conocimiento acabado e irrefutable, sino para facilitar y ayudar al grupo a compartir el conocimiento que tiene en su interior y a tomar del mundo nuevos conocimientos" (Gall 2005, pp. 2-3).

Para el caso particular de la I.E. Camilo Torres de Mocarí en Montería, las condiciones ambientales, sumadas a una baja capacidad de escucha de los estudiantes (de acuerdo a lo expresado por sus docentes), se configura como un factor que obstaculiza ese diálogo como fuente primaria para el desarrollo de relaciones pedagógicas que favorezcan y fortalezcan el proceso de enseñanza-aprendizaje. Desde esta dimensión, es más que pertinente revisar estas dos condiciones que se configuran también en mediadores de las prácticas y de las actividades escolares. Su función está en directa relación con los contenidos organizados en estrategias pedagógicas previamente determinadas, de ahí la preponderancia de definir bajo criterios pedagógicos el uso de los medios-recursos, pues su inmersión en el micromundo del aula de clases debe estar claramente articulado e integrado desde la propuesta curricular de la institución.

Se evidencia así que las relaciones pedagógicas, en el marco de esta realidad escolar, se siguen sustentado en la oralidad mediante la cual el docente verbaliza el conocimiento a través de esta forma de expresión, lo que finalmente no está favoreciendo la capacidad de escucha de los educandos, ni el uso de los medios-recursos, que queda bajo el dominio del habla como principal forma de lenguaje. Situación educativa que, como afirma Gall (2005), no contribuye a que los conocimientos puedan ser útiles en la transformación del mundo y de las relaciones sociales que lo conforman.

Son entonces las relaciones pedagógicas, en el marco de la articulación entre comunicación-educación, las que facilitan "la producción colectiva de conocimientos que genera nuevas herramientas (conceptuales, valorativas, técnicas, conductuales, etc.) que ayudan a modificar las prácticas y a visualizar las múltiples maneras en que se producen relaciones" (Gall, 2005).

\section{Lenguaje audiovisual}

Para el abordaje de esta dimensión y sus conclusiones, se tendrá como horizonte conceptual los postulados que propone Gutiérrez (2010) en su texto Creación multimedia y alfabetización en la era digital, cuyo presupuesto teórico parte de comprender que, a diferencia de lo que ocurre en la vida cotidiana de los estudiantes, las instituciones educativas y sus docentes centran el proceso de enseñanza desde el lenguaje verbal: "cuando todavía no se ha extendido suficientemente la alfabetización audiovisual en los planes de estudio, la forma predominante de estructurar y transmitir la información, se está mo- 
dificando hacia lo que podemos llamar el lenguaje multimodal y multimedia" (Gutiérrez, 2010, p. 171).

En esta tensión entre tradición y nuevos medios, los estudiantes de la Institución Educativa Camilo Torres de Montería, tienen la percepción que el uso de dichos recursos no facilita la comunicación entre ellos y el docente. Podría constituirse en motivo de disyuntiva -entre el medio-recurso y las formas de comunicación-diálogo-, el hecho de que los profesores tienen una marcada tendencia hacia el uso de recursos tradicionales como lo son el tablero, los libros, las carteleras, las revistas, los mapas y papelógrafos, instrumentos que parecen acrecentar las diferencias generacionales entre educadores y educandos.

De manera pedagógica - desde el lenguaje audiovisual-, se evidencia la necesidad de diseñar recursos que superen la oralidad como expresión privilegiada en el aula de clases para explorar otras alternativas (como ocasionalmente algunos docentes lo hacen a través de blogs, wikis, cine y páginas web), de forma tal que estudiantes y docentes no solo valoren el uso creativo que se le da a los mismos, sino que estratégicamente aborden lenguajes "multimodales y multicódigos que por sus propias características sirven de encuentro e integración de otros lenguajes como el verbal y el audiovisual" (Gutiérrez, 2010, p. 172).

\section{Mediaciones comunicativas}

Para el afinamiento de este indicador y las conclusiones que de él se derivan, se toma como orientador conceptual el texto La vida en las aulas, en el que se caracteriza la inmediatez del mundo escolar a través de signos transitorios en los que el educador deposita su confianza para decidir sobre los contenidos, las metodologías, los apoyos y demás recursos que se supone hacen eficientes los actos de enseñanza, inmersos, según el mismo autor, en modelos convencionales de racionalidad representados en el quehacer docente (Jackson, 1992).

En correspondencia con esta postura teórica, la fundamentación propia de esta investigación revalidó el concepto de mediación comunicativa en la praxis de la institución educativa sujeto de estudio. Desde esta realidad educativa, se logró comprender que las mediaciones comunicativas se potencializan haciendo uso de medios-recursos novedosos como las TIC, pues estas herramientas de apoyo facilitan comunicar al docente con los estudiantes en el marco de los contenidos temáticos a desarrollar en cada área de conocimiento. Situación pedagógica que se hace más asertiva cuando el profesor logra concebir al medio-recurso articulado con los procesos comunicativos inmersos al contexto del aula de clases y, en consecuencia, con las posibilidades formativas del mismo.

Pero si bien este reconocimiento contextual y formativo nutre las posibilidades para el desarrollo de las mediaciones comunicativas, la ausencia de un sistema de evaluación de los recursos-medios, crea una fisura teórico-práctica que va en detrimento del proceso de enseñanza-aprendizaje. Poder evaluar los valores implícitos, su estructura, funciones e impacto en el medio escolar, son más que una selección de necesidades por investigar, 
que incluso podrían generar claves para indagar sobre la ausencia de participación activa y crítica de los estudiantes a partir de las actividades que acompañan, como afirma Jackson (1992), a los procesos de pensamiento racional: "antes y después de su encuentro cara a cara con sus alumnos, el profesor a menudo parece ocuparse de un tipo de actividad intelectual que tiene muchas de las propiedades formales de un procedimiento para resolver problemas" (Jackson 1992, p. 173).

\section{Validación pedagógica}

La última conclusión se enfoca en la validación de los recursos audiovisuales como una iniciativa pedagógica. Como producto del trabajo de campo orientado por los instrumentos y su metodología, el grupo objeto de estudio (estudiantes del grupo 11-3) realizó tres videos, tres productos sonoros y un blog, luego de las etapas de socialización, capacitación, planeación y realización.

El indicador que sustenta la validez pedagógica de la propuesta se comprende en que una vez superada la alfabetización y uso instrumental de los medios, se hizo toda una propuesta audiovisual, sonora y de TIC, desde las formas expresivas que cada recurso ofrece por sus lenguajes y modos de comunicar. Como diría Cabero (1998), antes de encender una cámara o un micrófono, el grupo pensó en función de cada medio a utilizar, para quién iban dirigido los contenidos y que intención comunicativa querían desarrollar. Además, fueron temáticas trabajadas en el contexto de su realidad, no solo escolar, sino fundamentalmente, social, cultural y económica. Los jóvenes-estudiantes-realizadores, comprendieron, desde sus propias convicciones y criterios, que los medios audiovisuales son sistemas simbólicos cuya información genera en sus interlocutores diversidad de efectos cognitivos y pragmáticos, incluido el desarrollo de habilidades y competencias para ellos mismos.

Al hacer la transformación de una información escrita (textos sobre la moral y la ética en Platón) en recursos audiovisuales, los jóvenes-estudiantes-realizadores reconocieron y llevaron al ejercicio práctico las seis funciones que pueden desempeñar las TIC en la enseñanza, según Rodríguez Neira (1996):

- Al conjugar video, radio y blog, fortalecieron sus procesos cognitivos a través del uso de sistemas multimediáticos que los llevaron a explorar todos sus sentidos para la adquisición de conocimiento: los textos consultados sobre ética y moral en Platón, se transfiguraron en propuestas comunicativas que giraron en torno a temáticas como el embarazo no deseado, la corrupción en la educación, la drogadicción, la adopción entre iguales y el suicidio.

- Lograron superar las fronteras del espacio-tiempo propias del aula de clases, en virtud de las exigencias que cada medio les generó.

- Pudieron ampliar su experiencia personal más allá de sus docentes y de su institución educativa a través de las experiencias en otros espacios (la calle, la universidad, entre 
otros) y con otros sujetos (profesores y estudiantes de universidad).

- Desarrollaron nuevos sistemas de codificación y decodificación articulados a la diversidad de mensajes mediados en soportes sonoros, audiovisuales, informáticos y de TIC en general.

- Crearon nuevas relaciones en de la institución, pues desde su rol de realizadores asumieron el perfil de capacitadores de otros jóvenes de diferente nivel escolar.

- Reconocieron la influencia de los recursos sonoros y audiovisuales en la relación realidad-ficción, con la realización de diversos formatos como puestas en escena de dramatizados, entrevistas, videoclips, radio revistas y el diseño y montaje de un blog.

Finalmente, se puede concluir que la implementación de recursos audiovisuales como estrategia de validación pedagógica logró priorizar la selección de la información en función del diseño de cada medio y de las necesidades e intereses de la comunidad académica. Además, se generaron nuevas narrativas (audiovisuales y sonoras) diferentes a las textuales y orales propias del curso y de la temática. Sin embargo, y a pesar que las etapas le dieron un norte a la planeación para realizar cada medio-recurso, los tiempos en las instituciones educativas no se cumplen por factores externos, como los paros de docentes, las reuniones y hasta por problemas de inundación, variable que hace más complejo el proceso. Pero lo más destacable fue que la implementación de los videos, los productos sonoros y el blog, mediaron los valores implícitos de cada recurso con los valores preponderantes del contexto escolar.

\section{Referencias}

Blázquez, F. (1994). Propósitos formativos de las nuevas tecnologías de la información y la comunicación en la formación de maestros. En F. Blázquez, J. Cabero y F. Loscertales (Eds.), Nuevas tecnologías de la información y la comunicación (pp. 257-268). Sevilla: Alfar.

Cabero, J. (1998). Usos e integración de los medios audiovisuales y las nuevas tecnologías en el currículum. Recuperado de http://ardilladigital.com/DOCUMENTOS/TECNOLOGIA\%20EDUCATIVA/ TICs/T2\%20NNTT\%20Y\%20N\%20ED/CABERO\%20NNTT\%20Y\%20CURRICULUM.pdf

Crovi, D. (2010). El entramado reticular de la educación: una mirada desde la comunicación. En R. Aparici (Coord.), Educomunicación: más allá del 2.0 (pp. 105-128). Barcelona: Gedisa.

Díaz, E., Alvarino, G. y Carrascal, N. (2011). Enfoques de aprendizaje y niveles de compresión, El ambiente universitario en ambientes tecnológicos. Montería: SUE Caribe. Recuperado de https://issuu.com/ librosisabel/docs/enfoques_aprendizaje_niveles_compresi_n

Escudero, J. (1995). La integración de las nuevas tecnologías en el currículum y en el sistema escolar. En J. L. Rodríguez Diéguez y Ó. Sáenz (Coords.), Tecnología educativa: nuevas tecnologías aplicadas a la educación (pp.397-412). Navarra: Marfil.

Freire, P. (1973). ¿Extensión o comunicación? La concientización en el medio rural. Buenos Aires: Siglo XXI.

Gall, E. (2005). Prácticas educomunicativas: miradas sobre lo inacabado. Recuperado de https://www. 
usp.br/nce/?wcp=/aeducomunicacao/saibamais/textos/texto, 2,46,268

Gutiérrez, A. (2010). Creación multimedia y alfabetización en la era digital. En R. Aparici (Coord.), Educomunicación: más allá del 2.0 (pp. 171 186). Barcelona: Gedisa.

Hernández, R., Fernández, C. y Baptista, P. (1999). Metodología de la Investigación. México: McGraw Hill. Jackson, P. (1992). La vida en las aulas. Madrid: Morata.

Mallas, S. (1979). Medios audiovisuales y pedagogía activa. Barcelona: CEAC.

Marrero, J. (1993). Las teorías implícitas del profesorado: vínculo entre la cultura y la práctica de la enseñanza. En M. J. Rodrigo, A. Rodríguez y J. Marrero (Eds.), Las teorías implícitas: una aproximación al conocimiento cotidiano (pp. 243-276). Madrid: Visor.

Porcher, L. (1974). La escuela paralela. Buenos Aires: Kapelusz.

Rodríguez, N. (1996). Modelos y medios. Aula Abierta, (66). 3-30.

Salinas, J. (1987). Mass-media y educación social. en A. Colom (Ed.), Modelos de intervención socioeducativa (pp. 252-276). Madrid: Narcea. 